\title{
Reduction of Perineal Evoked Excitatory Postsynaptic Potentials in Cat Lumbar and Sacral Motoneurons during Micturition
}

\author{
B. Fedirchuk, ${ }^{1, a}$ J. W. Downie, ${ }^{2}$ and S. J. Shefchyk ${ }^{1}$ \\ 'Department of Physiology, University of Manitoba, Winnipeg, Canada R3E OW3 and ${ }^{2}$ Department of Pharmacology, \\ Dalhousie University, Halifax, Nova Scotia, Canada B3K 3E7
}

These experiments were undertaken to examine whether both premotoneuronal mechanisms and direct actions on motoneurons could contribute to suppression of excitatory perineal reflex pathways during micturition. Intracellular recordings were obtained from motoneurons innervating the external urethral sphincter (EUS), external anal sphincter (EAS), and selected hindlimb muscles in decerebrate male cats. The peak amplitudes of EPSPs evoked by electrical stimulation of peripheral cutaneous afferents were measured during micturition. In the EUS, EAS, and hindlimb motoneurons examined, EPSPs produced by stimulation of perineal afferents (superficial perineal or sensory pudendal nerves) were reduced in amplitude during micturition. The sample of PSPs evoked by stimulation of hindlimb cutaneous nerves recorded in hindlimb motoneurons revealed that these PSPs could also be reduced. In contrast, no changes were seen in monosynaptic EPSPs evoked by muscle afferent stimulation.

The present study demonstrates that during micturition there is a strong suppression of perineal reflexes to both sphincter and hindlimb motoneurons. Since reduced EUS activity is required for efficient micturition, suppression of the strong excitatory perineal input to EUS motoneurons likely contributes to decreased EUS activity during the bladder contraction. It appears that the micturition circuitry utilizes both premotoneuronal mechanisms and direct motoneuronal inhibition to achieve this reflex suppression. The function of the micturition-related reduction of perineal reflexes to hindlimb or EAS motoneurons is not known at this time and further investigations are required to elucidate the interaction between micturition circuitry and hindlimb cutaneous pathways.

[Key words: sacral, pudendal, urethra, bladder, spinal reflexes]

It has been shown previously that external urethral sphincter (EUS) and external anal sphincter (EAS) motoneurons receive strong polysynaptic excitation from sensory pudendal (SPud)

\footnotetext{
Received Oct. 14, 1993; revised Feb. 25, 1994; accepted Apr. 13, 1994.

This work was funded by an operating grant from the Medical Research Council (MRC) of Canada to S.J.S.; B.F. is funded by the MRC studentship program. We thank Sharon McCartney and Maria Setterbom for their excellent technical assistance and Dr. Brian Schmidt for his helpful comments.

Correspondence should be sent to S. J. Shefchyk, Department of Physiology, University of Manitoba, 770 Bannatyne Avenue, Winnipeg, Canada R3E 0W3.

aPresent address: Institute of Neurophysiology, The Panum Institute, Blegdamsvej 3C, University of Copenhagen, DK-2200 Copenhagen N, Denmark.

Copyright (C) 1994 Society for Neuroscience $0270-6474 / 94 / 146153-07 \$ 05.00 / 0$
}

and superficial perineal (SPeri) afferents (Fedirchuk et al., 1992a). In contrast, there are only weak synaptic effects from other cutaneous hindlimb afferents onto sphincter motoneurons. While it has been hypothesized that tonic activity in the EUS during continence is produced by increased bladder afferent activity during bladder distention (see deGroat, 1990), sensory information carried in the pudendal and perineal nerves also contributes to both tonic and phasic activity of the EUS (Garry et al., 1959; B. Fedirchuk and S. J. Shefchyk, unpublished observations). During micturition, tonic and phasic reflex activity of the EUS is normally decreased (Rampal and Mignard, 1975; Fedirchuk and Shefchyk, 1993). Sethi et al. (1989) noted that in spinal cord patients displaying bladder-sphincter dyssynergia, excitatory perineal to sphincter reflexes such as the bulbocavernosus reflex are not suppressed during bladder contractions. Evidently, suppression of tonic and phasic EUS activity is required in man and cat in order to facilitate efficient bladder emptying during micturition.

There are several mechanisms that may contribute to the suppression of urethral sphincter activity during micturition. Previous studies have demonstrated that EUS motoneurons hyperpolarize during micturition (Shimoda et al., 1992; Fedirchuk and Shefchyk, 1993). This hyperpolarization was associated with an increase in motoneuronal membrane conductance and was reversed with injection of chloride into the motoneuron (Fedirchuk and Shefchyk, 1993). This latter study concluded that EUS motoneurons received postsynaptic inhibition during micturition and this inhibition was restricted to EUS motoneurons since EAS motoneurons did not hyperpolarize.

While postsynaptic events occurring at the motoneuron may contribute to reflex suppression, premotoneuronal mechanisms such as inhibition of excitatory interneurons or primary afferent depolarization (PAD) may also alter reflex transmission. The present study examines whether there is evidence for premotoneuronal mechanisms contributing to suppression of perineal reflexes during micturition. In addition, the study tests whether this reflex suppression is focused only onto perineal pathways to EUS motoneurons or whether perineal reflexes to other motoneurons are also affected. This possibility was addressed by obtaining intracellular recordings from EUS, EAS, and hindlimb muscle motoneurons and assessing the effect of the central micturition circuitry on excitation or inhibition evoked by perineal afferents. Since EUS and EAS motoneurons receive only weak inputs from hindlimb cutaneous and muscle afferents (Fedirchuk et al., 1992a), the ability of the micturition circuitry to alter these pathways was assessed in hindlimb muscle motoneurons. 


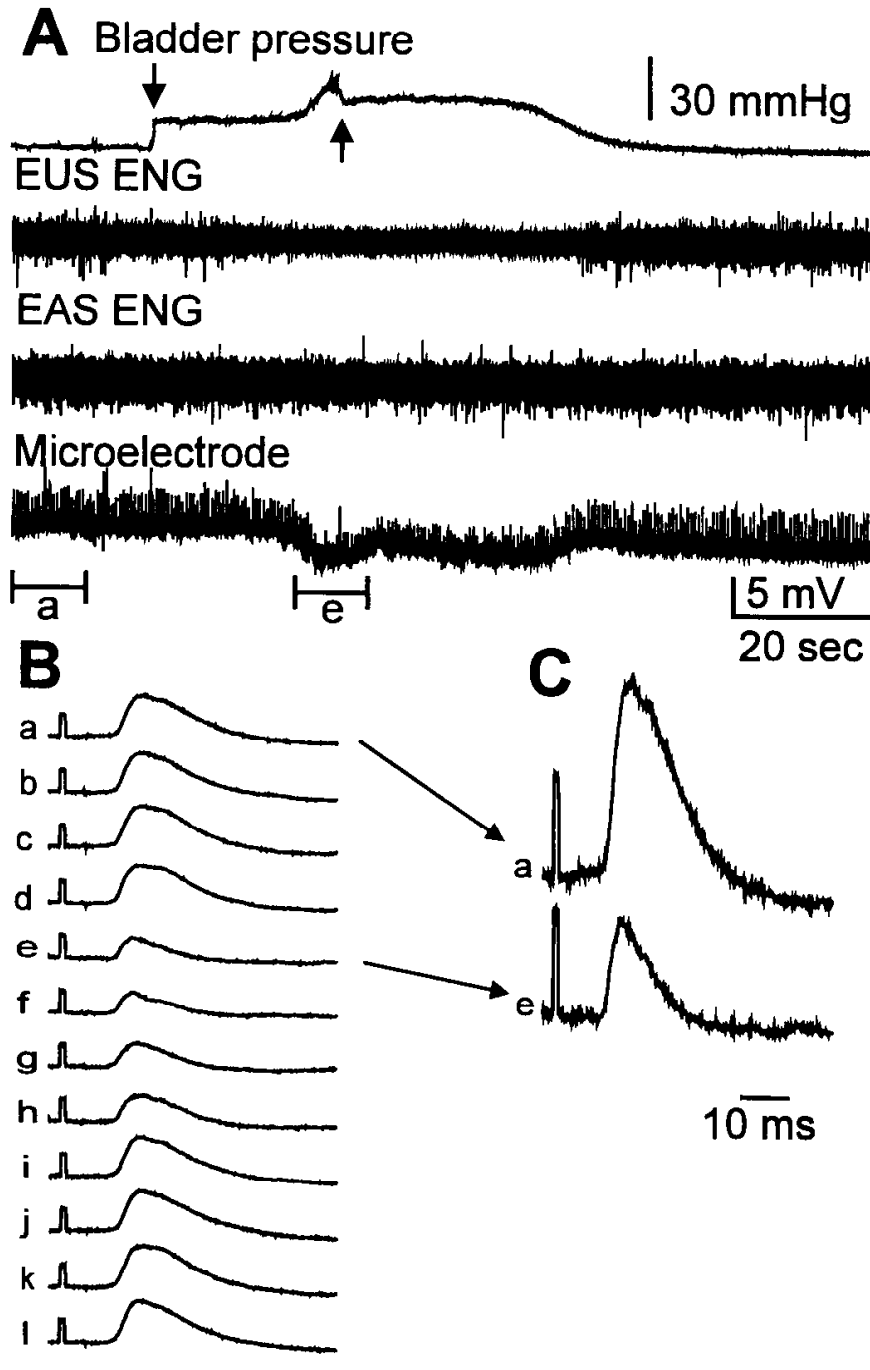

Figure 1. Intracellular recording from an EUS motoneuron (resting $E_{m} \sim-70 \mathrm{mV}$ ) during an isometric bladder contraction. In $A$ records of bladder pressure (arrows indicate the onset and offset of infusion), EUS ENG, EAS ENG, and EUS motoneuron membrane potential (5 $\mathrm{mV}$ calibration) are shown with SPud nerve stimulation (1.7T). The EPSPs produced were averaged; each average was obtained during consecutive $10 \mathrm{sec}$ periods of $A(B ; 19$ sweeps per average; calibration pulses, $2 \mathrm{mV}$ ). The averaged EPSP preceding micturition $(a)$ and the smallest EPSP of this trial ( $e$; amplitude $51 \%$ of $a$ ) are shown enlarged in $C$ (calibration pulse, $2 \mathrm{mV}$ ). Time calibration in $C$ also applies to $B$.

Portions of this study have been presented previously in abstract form (Fedirchuk et al., 1991).

\section{Materials and Methods}

Data were obtained from 20 decerebrate male cats weighing between 2.1 and $3.3 \mathrm{~kg}$. Halothane delivered in a mixture of oxygen and nitrous oxide was used to induce ( $5 \%$ halothane) and maintain anesthesia (0.8$1.5 \%)$ throughout surgery until the decerebration was completed. The decerebration involved removal of the cerebral cortex followed by a precollicular postmammillary transection of the midbrain. All remaining tissue rostral to the transection was removed. The arterial blood pressure was monitorcd via a cannula inserted in the right femoral artery and dextran (Gentran; 6\%) was administered through venous cannula as required to replace blood volume. A continuous infusion of a glucose/ sodium bicarbonate buffer solution $\left(0.84 \mathrm{gm}\right.$ of $\mathrm{NaHCO}_{3}$ and $5 \mathrm{gm}$ of glucose $/ 100 \mathrm{ml}$ ) was administered $(5 \mathrm{ml} / \mathrm{hr})$. The animal's core temperature was maintained at $37-38^{\circ} \mathrm{C}$. Paralysis was achieved by the administration of gallamine triethiodide $(5 \mathrm{mg} / \mathrm{hr})$, and the animal was artificially ventilated. Expired $\mathrm{CO}_{2}$ was maintained at $3-4 \%$.
A lateral incision starting at the base of the tail and extending down the hindlimb exposed the ischiorectal cavity and various nerve branches along the sciatic nerve. Branches of the ipsilateral pudendal nerve innervating the EUS muscle, the EAS muscle, and the sensory branch of the pudendal nerve (SPud) were dissected from surrounding tissue and cut. Other cutaneous and muscle nerves that were dissected and cut included: cutaneous superficial perineal (SPeri), caudal cutaneous femoralis (CCF), caudal cutaneous sural (CCS), posterior biceps and semitendinosus (PBSt), and medial gastrocnemius (MG). A mineral oil pool was fashioned from hindlimb skin and maintained at approximately $38^{\circ} \mathrm{C}$. Each nerve was placed on silver bipolar electrodes that could be used for either stimulating or recording. A laminectomy exposed the L6 to 33 spinal cord and the animal was placed in a spinal cord recording frame. Peripheral nerve stimulus strengths were expressed in multiples of the threshold $(T)$ current (constant current, $0.2 \mathrm{msec}$ pulses) that produced a volley that was just detectable with a cord dorsum recording electrode.

Micturition was evoked by infusion of saline $\left(35^{\circ} \mathrm{C} ; 2 \mathrm{ml} / \mathrm{min}\right)$ into the bladder via a suprapubic catheter. Micturition threshold volume ranged from 6 to $30 \mathrm{ml}$. At threshold volume, bladder pressure increased, EUS electroneurographic (ENG) activity decreased, and fluid in the bladder was expelled in a continuous stream. In seven cats, the bladder neck was ligated and infusion of fluid into the bladder continued until periodic isometric bladder contractions began. Infusion was then stopped and periodic contractions (1-2 per min) continued as long as the volume in the bladder was maintained. Peak pressure during each of these isometric micturition contractions was comparable to, or greater than, peak pressures measured in freely voiding preparations. EUS ENG activity decreased during each isometric bladder contraction.

Intracellular records were obtained using glass microelectrodes filled with 2 м potassium citrate (tip diameter, 1.6-1.8 $\mu \mathrm{m}$; resistance, 2-5 $\mathrm{M} \Omega$ ). Motoneurons were impaled in L7 and $\mathrm{S} 1$ spinal segments and identified using antidromic activation from one of the peripheral nerves. Bladder pressure, ENGs, and stimulus markers were digitized and stored on computer (Masscomp 5400). Low gain and high gain intracellular microelectrode records were digitized at rates of 5 and $15 \mathrm{kHz}$ respectively. Each trial typically consisted of a prevoid, voiding, and postvoid period. Throughout each trial a peripheral nerve was stimulated at a frequency from 0.5 to $3.5 \mathrm{~Hz}$. Sometimes excitatory postsynaptic potentials (EPSPs) evoked from different peripheral nerves were examined during the same trial. This was achieved by alternating the stimulus between two or more different nerves throughout the trial. The cord dorsum potentials evoked by the nerve stimuli were evaluated and interstimulus intervals chosen to ensure that no response was affected by the stimulus preceding it (e.g., through presynaptic inhibition; see Angel et al., in press). The voiding cycle was divided into time segments and intracellular responses evoked by stimulation of a particular peripheral nerve during each period were averaged (5-49 sweeps). EPSP pcak amplitudes wcre measured from averaged records, and averages from various time segments within a trial were compared. The peak amplitude of the averaged EPSP either preceding or following the bladder contraction was used as the control amplitude within each trial. EPSP amplitudes are expressed as percentage of the control value. References to EPSP changes refer to peak amplitude only. In some cases, peripheral nerve stimulation evoked pure IPSPs. The amplitude changes of these IPSPs during micturition were assessed as has been described for EPSPs. In cases where peripheral nerve stimulation evoked a mixed excitatory and inhibitory PSP (E/I PSP) in the motoneuron, the amplitude of the early EPSP was difficult to determine with confidence because the IPSP began near, or at the peak of the EPSP. While we report the incidence of these mixed E/I PSPs in the motoneurons sampled, amplitudes of the excitatory component of mixed E/I PSPs were not included with the sample of pure PSPs examined during micturition.

\section{Results}

\section{EUS motoneurons}

Intracellular recordings were obtained from 22 EUS motoneurons (mean resting $E_{m},-50 \mathrm{mV}$ ). Electrical stimulation of SPud or SPeri nerves produced EPSPs ranging from 1.6 to $4.5 \mathrm{mV}$ (also see Fedirchuk et al., 1992a). Pure IPSPs were not evoked by SPud or SPeri stimulation and in only 1 of 22 motoneurons did SPud evoke a mixed E/I PSP. The central latencies ranged 

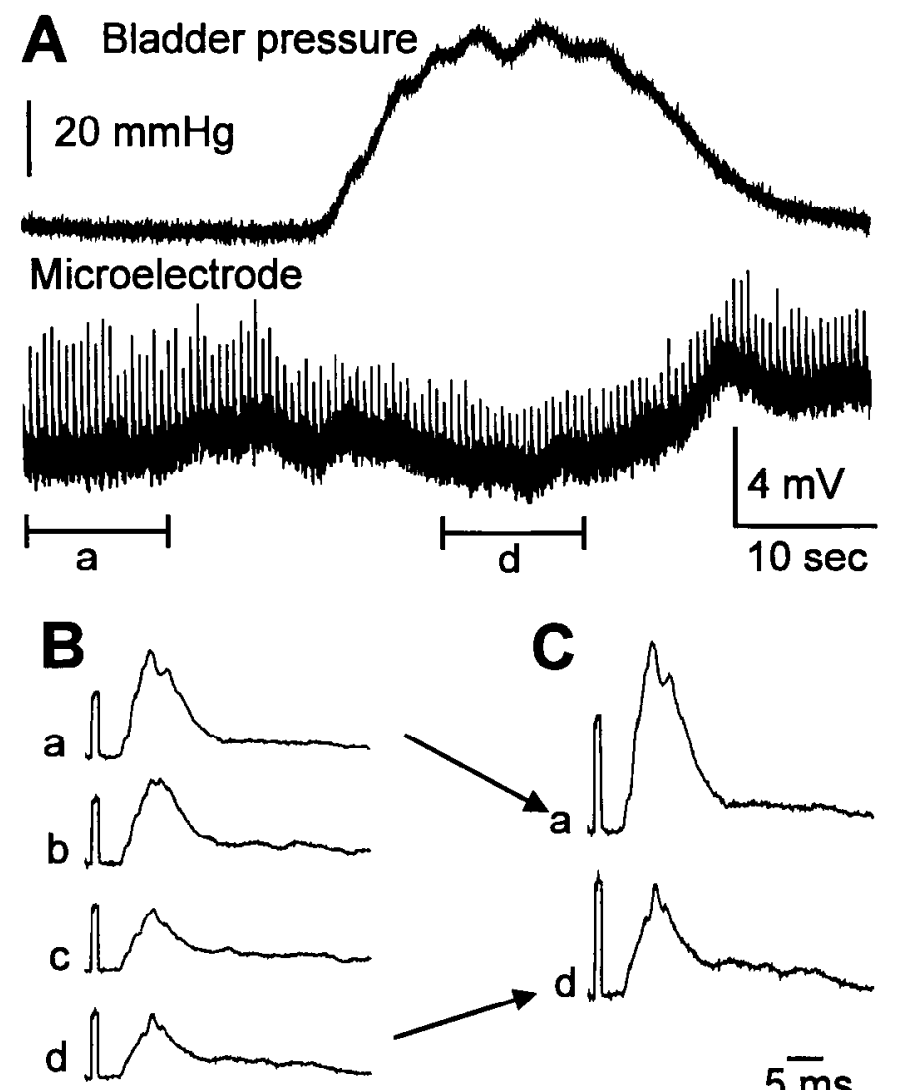

d
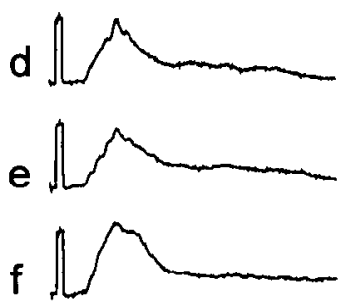

Figure 2. Intracellular recording from an EUS motoneuron (resting $E_{m} \sim-45 \mathrm{mV}$ ) during an isometric bladder contraction $(A)$ and electrical stimulation of the SPeri nerve at 5T. Averaged SPeri-evoked EPSPs shown in $B(a-f)$; each average (20 sweeps) was obtained during consecutive $10 \mathrm{sec}$ time periods of $A$. An averaged EPSP preceding micturition $(a)$ and that with the smallest amplitude during micturition $(d)$ are shown in $C$.

from 2.6 to $6.2 \mathrm{msec}$ for EPSPs evoked by SPud stimulation and from 1.9 to $2.5 \mathrm{msec}$ for EPSPs evoked by SPeri stimulation.

Figure $1 A$ shows the typical response of an EUS motoneuron recorded during micturition. The motoneuron hyperpolarized $5 \mathrm{mV}$ (resting $E_{m},-70 \mathrm{mV}$ ) during the isometric bladder contraction and decreased EUS ENG activity. During this hyperpolarization, the EPSP produced by electrical stimulation of the SPud nerve (1.7T) was reduced to $51 \%$ of that prior to micturition (see Fig. 1B,C). The time course of EPSP reduction coincided with EUS motoneuron hyperpolarization. In 16 EUS motoneurons in which EPSPs were evoked by SPud stimulation (1.1-11T) during micturition, EPSPs ranged from 0 to $116 \%$ of control (mean $=41 \%$ ). In 13 of 16 motoneurons SPud-evoked EPSPs were dramatically reduced (i.e., were $<60 \%$ of control) while in the remaining three motoneurons the EPSPs were $90 \%$, $96 \%$, and $116 \%$ of control. Even with these three trials included in the sample, the reduction in EPSPs observed during micturition was statistically significant (paired sign test, $p<0.001$ ).

Both EPSPs produced by low $(\leq 2 \mathrm{~T})$ and high $(\geq 10 \mathrm{~T})$ SPud nerve stimulation strengths were reduced during micturition.

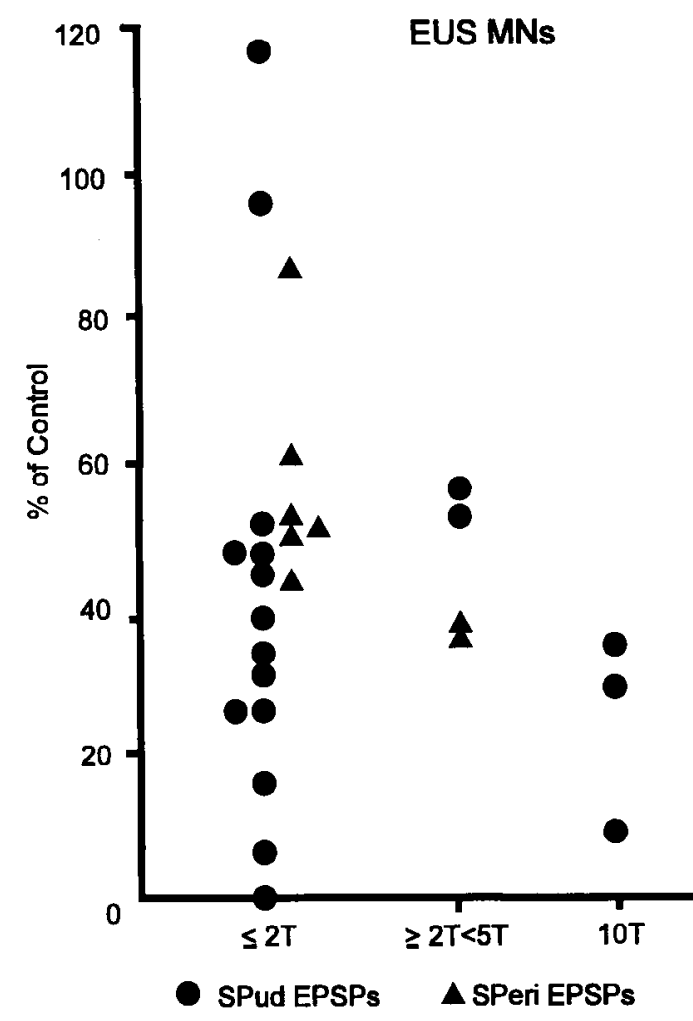

Figure 3. Summary of the relative amplitudes of SPud-evoked and SPeri-evoked EPSPs in EUS motoneurons during micturition. EPSP amplitudes are expressed as percentage of the control EPSP amplitude for each trial.

Unfortunately, examination of changes in amplitudes of EPSPs evoked by a wider range of stimulus strengths could not be done routinely because of the propensity of higher stimulation strengths to evoke action potentials. In five additional motoneurons, SPud nerve stimulation (1.4-11T) produced action potentials that, in each case, ceased during micturition and resumed following the bladder contraction. These observations show the ability of micturition to suppress reflex-evoked discharges in these motoneurons.

Figure 2 illustrates an example of the reduction of the SPerievoked EPSP recorded in an EUS motoneuron during micturition. The EPSPs $(1.2-5 \mathrm{~T} ; n=10)$ were significantly reduced (paired sign test, $p<0.01$ ), ranging from $36 \%$ to $87 \%$ (mean $=$ $56 \%$ ) of control values. Similar to observations for SPud-evoked EPSPs, SPeri-evoked EPSPs were smallest when the EUS motoneuron hyperpolarized. The reduction of both SPud and SPerievoked EPSPs did not have a gradual onset related to the degree of bladder filling prior to micturition. Rather, decreases in EPSP amplitude coincided with the bladder contraction and decrease in EUS ENG. Figure 3 summarizes the amplitudes of EPSPs recorded in EUS motoneurons evoked by stimulation of SPud or SPeri nerves during micturition expressed as percentage of control amplitude.

\section{EAS motoneurons}

Intracellular recordings were obtained from 15 EAS motoneurons (mean resting $E_{m},-49 \mathrm{mV}$ ). Similar to EUS motoneurons, inputs from SPud and SPeri afferents are generally excitatory (EPSP amplitudes, 0.7-6.0 mV; latencies, 2.1-5.4 msec for SPud, 


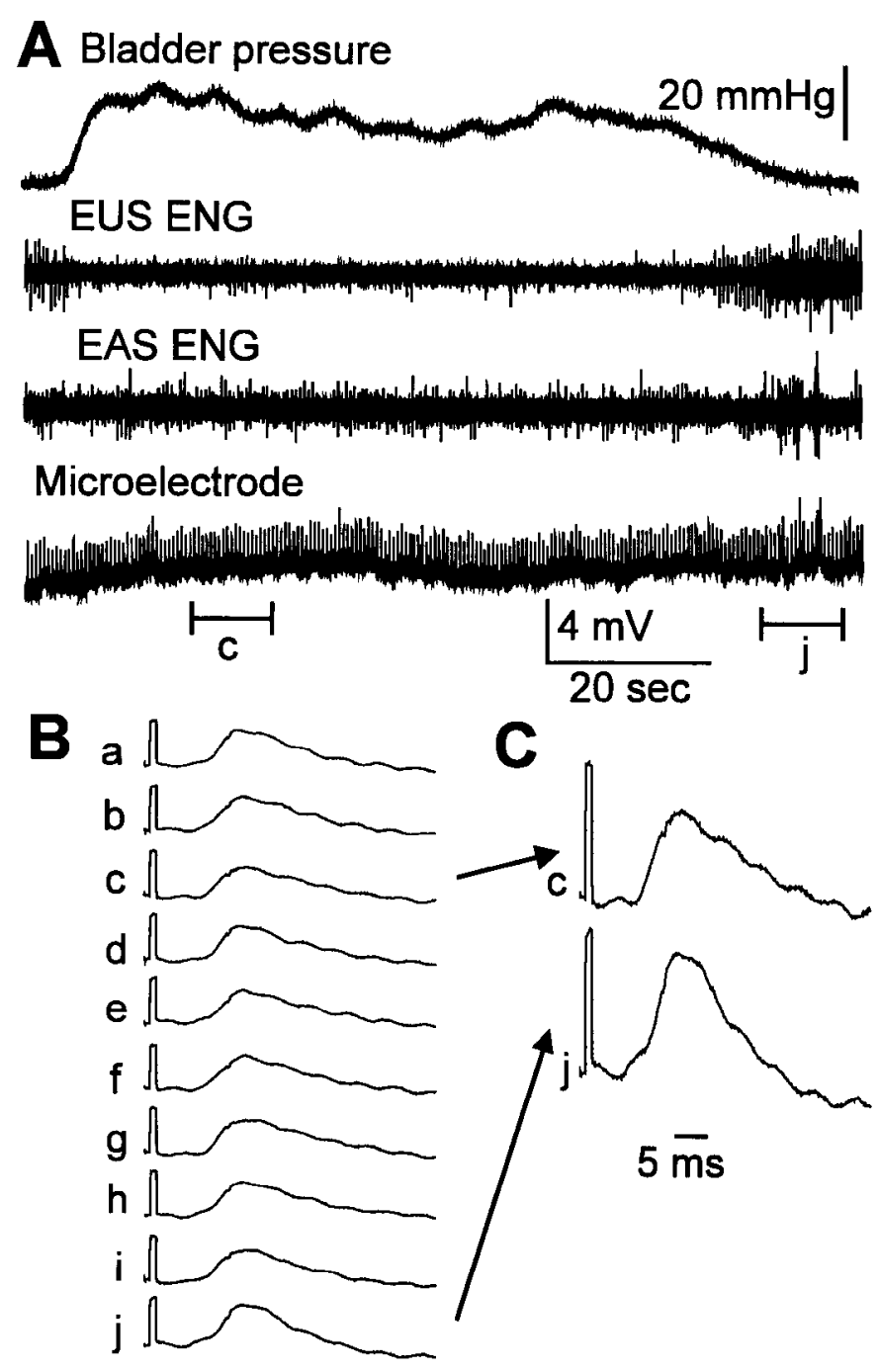

Figure 4. Intracellular recording from an EAS motoneuron (resting $E_{m}$ $-63 \mathrm{mV}$ ) during an isometric bladder contraction and SPud stimulation at 1.1T $(A) . B$ illustrates averaged EPSPs $(a-j)$ obtained during the trial; each average is comprised of 20 sweeps obtained from consecutive 10 sec time periods of $A$. The averaged EPSP for the control period, in this case following the bladder contraction $(j)$, and the smallest averaged EPSP seen during micturition $(c)$ are shown enlarged in $C$.

2.4-3.1 msec for SPeri). In four EAS motoneurons, SPud stimulation evoked E/I PSPs (see also Fedirchuk et al., 1992a).

Figure $4 A$ illustrates a typical EAS motoneuron in which the motoneuron membrane potential depolarized slightly during micturition (see also Fedirchuk and Shefchyk, 1993). Figure $4 B$ shows SPud-evoked (1.1T) EPSPs during this trial. The reduction of the EPSP amplitude (to $80 \%$ of control) is shown more clearly in Figure $4 C$. In 10 EAS motoneurons the SPud-evoked EPSPs $(1.1-5 \mathrm{~T})$ were reduced to $7-90 \%$ of control (paired sign test, $p<0.002$; mean $=73 \%$ ) during micturition. EPSPs evoked by stimulation of the SPeri nerve (1.4-2T) in five EAS motoneurons were reduced to $15-83 \%$ of control. Figure 5 summarizes the change in amplitude of EPSPs evoked by SPud and SPeri stimulation in EAS motoneurons. As with EUS motoneurons, a more rigorous comparison of reductions of EPSPs evoked by low $(\leq 2 \mathrm{~T})$ and high $(\geq 10 \mathrm{~T})$ stimulation strengths was limitcd because of the occurrence of stimulus-evoked action potentials in the EAS motoneurons sampled.

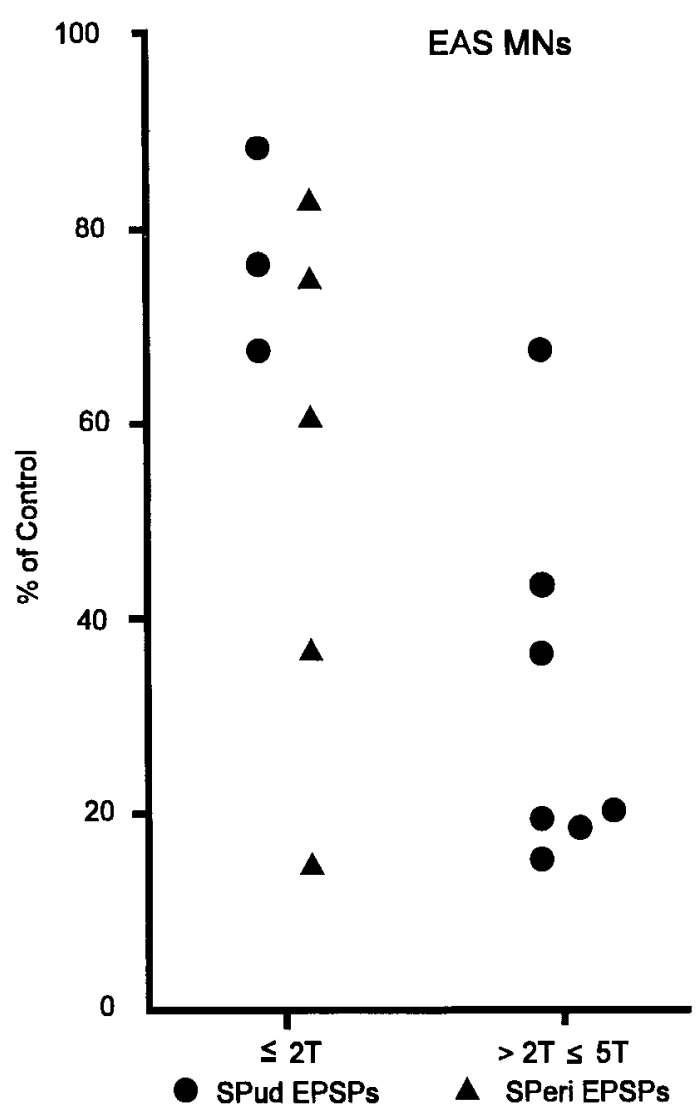

Figure 5. Summary of the relative amplitudes of EPSPs evoked by stimulation of SPud or SPeri nerves during micturition. The values are expressed as percentage of the control EPSP amplitude within each trial.

\section{Hindlimb motoneurons}

The possibility exists that a variety of reflex pathways, in addition to perineal reflexes to sphincter motoneurons, are suppressed during micturition. However, hindlimb cutaneous and muscle afferent pathways have only infrequent and weakly expressed actions on EUS and EAS motoneurons (Fedirchuk et al., 1992a). Therefore, to address this issue we sought to record from hindlimb motoneurons receiving input from both perineal and hindlimb afferent pathways. Motoneurons innervating PBSt and $\mathrm{MG}$ hindlimb muscles were found to occasionally receive synaptic input from SPud or SPeri afferents. However, higher stimulus strengths (2.5-20T) were required to elicit PSPs in these motoneurons in contrast to the low stimulation strengths usually effective for evoking EPSPs in EUS or EAS motoneurons.

Intracellular records were obtained from PBSt motoneurons (average resting $E_{m},-61 \mathrm{mV}$ ) and $\mathrm{MG}$ motoneurons (average resting $E_{m},-60 \mathrm{mV}$ ) that showed either a small transient depolarization or no membrane potential change during micturition. An example of such a recording in a PBSt motoneuron is shown in Figure 6 $\mathrm{A}$. In $10 \mathrm{PBSt}$ and three MG motoneurons SPud stimulation (2.5-20T) evoked 1.5-3.3 mV EPSPs with central latencies of $1.5-4.0 \mathrm{msec}$. In the other motoneurons, SPud stimulation evoked either a mixed E/I PSP $(n=4 \mathrm{PBSt}$, $3 \mathrm{MG}$ ) or a pure IPSP ( $n=4 \mathrm{PBSt}, 3 \mathrm{MG} ;-0.8$ to $-2.9 \mathrm{mV}$, 3.1-4.7 msec). When the trials assessing EPSP and IPSP amplitudes were pooled ( $n=20$ ), PSPs were smaller during micturition than control (paired sign test, $p<0.01$; mean $=63 \%$ of control; range, 32-153\%) despite three trials where EPSPs 
A

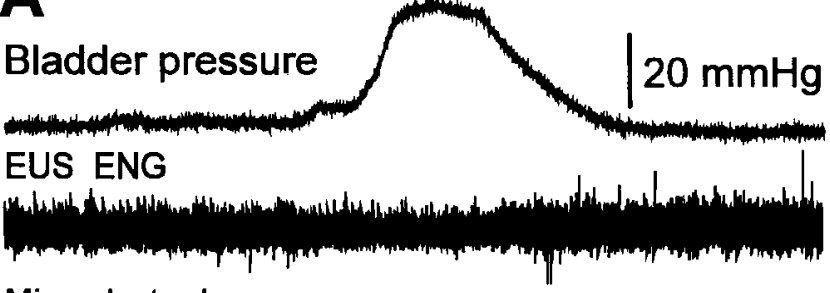

Microelectrode
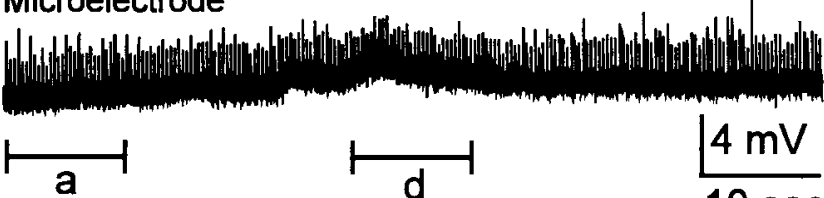

$4 \mathrm{mV}$

$10 \mathrm{sec}$
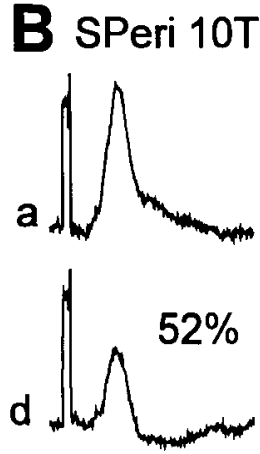

a
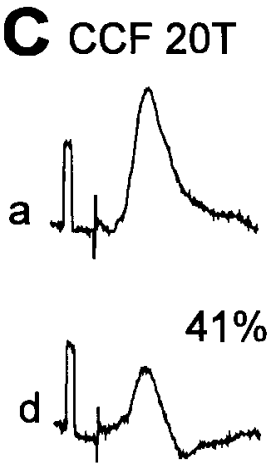

D PBSt 3.2T

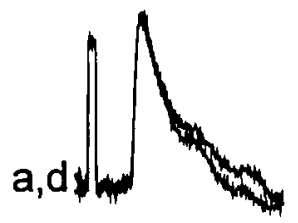

$5 \overline{\mathrm{ms}}$
Figure 6. Intracellular recording from a PBSt motoneuron (resting $E_{m}$, $-65 \mathrm{mV}$ ) during distention-evoked micturition. Averaged EPSPs produced by stimulation of SPeri (10T) and CCF (20T) are illustrated in $B$ and $C$, respectively. In both cases the amplitude of the averaged EPSP during micturition $(d)$ was reduced compared to that preceding micturition $(a)$. In this trial, an averaged homonymous monosynaptic EPSP (3.2T) was unchanged during micturition $(D)$. Calibration pulses in $B$ $D$ are $2 \mathrm{mV}, 2 \mathrm{msec}$; 10 sweeps per average. were larger during micturition. The amplitudes of the seven IPSPs ranged from 0 to $64 \%$ of control during micturition.

SPeri stimulation ( 5 or $10 \mathrm{~T}$ ) evoked EPSPs in four PBSt motoneurons (0.9-2.4 mV, 1.8-3.7 msec) and an IPSP in one PBSt motoneuron $(-1.1 \mathrm{mV}, 4.3 \mathrm{msec})$ that were assessed during micturition. Figure $6 B$ shows an example where the SPeri-evoked EPSP averaged during the bladder contraction is about half the size of the control EPSP. All of these SPeri-evoked PSPs were reduced during micturition and ranged from $24 \%$ to $83 \%$ of control.

CCS stimulation (1.2-20T) evoked EPSPs in four PBSt motoneurons and $5 \mathrm{MG}$ motoneurons $(1.3-5.9 \mathrm{mV}, 2.1-3.6 \mathrm{msec})$ and a pure IPSP in 1 PBSt motoneuron $(-3.4 \mathrm{mV}, 3.3 \mathrm{msec})$. Stimulation of CCF (5-20T) produced EPSPs in two PBSt motoneurons (1.9-2.7 mV, 1.3-2.3 msec), one of which is illustrated in Figure $6 C$. A pure IPSP $(-2.5 \mathrm{mV}, 7.5 \mathrm{msec})$ was evoked by CCF stimulation in one other PBSt motoneuron. Examination of the relative amplitudes of all pure EPSPs or IPSPs produced in PBSt and MG motoneurons from stimulation of either CCS or CCF nerves (data pooled, $n=13$ ) revealed that the amplitudes of these PSPs were also significantly reduced during micturition (paired sign test, $p<0.01$; mean $=72 \%$ of control). In an additional six PBSt motoneurons and one MG motoneuron, CCS or CCF stimulation evoked E/I PSPs that were not quantified during micturition. Figure 7 summarizes the relative amplitudes of PSPs evoked by stimulation of perineal (Fig. 7A) and hindlimb cutaneous (Fig. $7 B$ ) nerves recorded in PBSt and MG motoneurons during micturition.

In seven PBSt motoneurons, a monosynaptic homonymous EPSP was evoked (1.1 to 3.2T) throughout micturition. As shown in Figure $6 D$, the averaged monosynaptic EPSP was unchanged during micturition (control and micturition averages are shown superimposed). In five of six motoneurons where both monosynaptic and perineal-evoked PSPs were tested in the same trial, EPSPs evoked by stimulation of SPud or SPeri nerves were reduced during micturition while monosynaptic EPSPs were
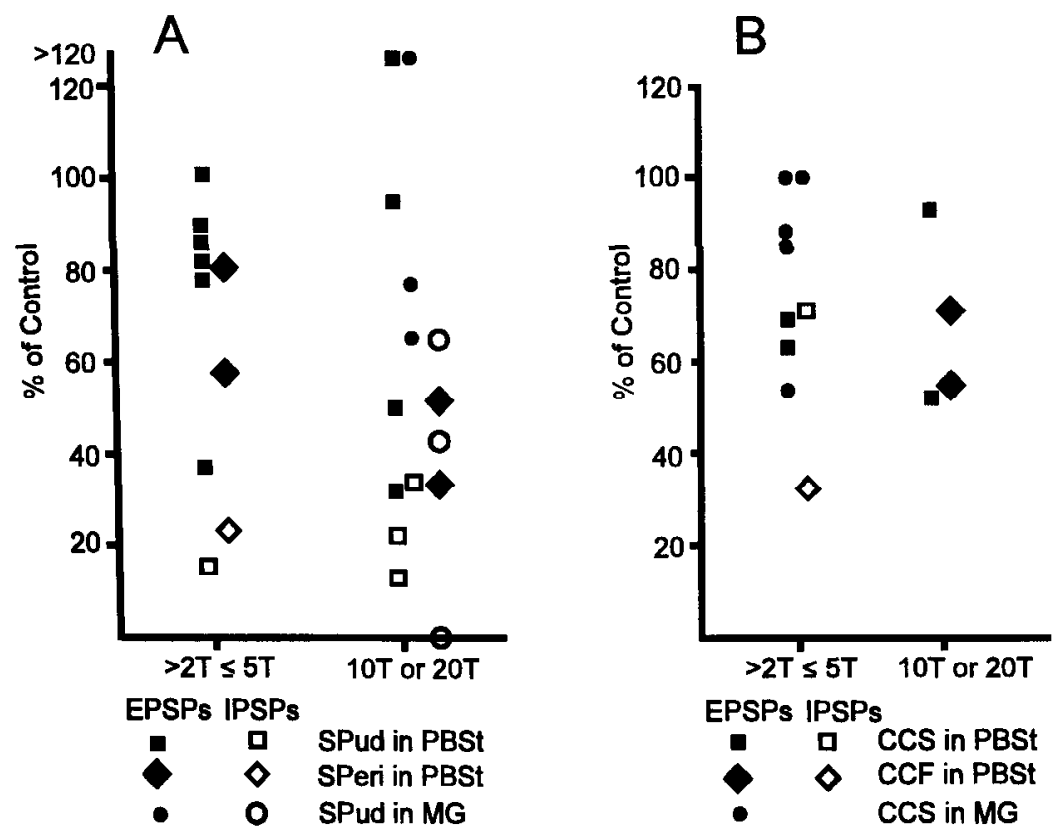

Figure 7. Summary of relative amplitudes of PSPs evoked by stimulation of SPud and SPeri nerves $(A)$, or CCF and $\operatorname{CCS}(B)$ nerves during micturition in PBSt and MG motoneurons. All PSP amplitudes are expressed as percentage of the control amplitude within each trial. 
unaffected. In the other motoncuron, neither the SPud EPSP (20T) nor the monosynaptic EPSP changed amplitude during micturition.

\section{Discussion}

This study has documented a decrease in the amplitude of EPSPs evoked by perineal (SPud and SPeri) afferent stimulation in urethral sphincter, anal sphincter, and hindlimb motoneurons during micturition. PSPs evoked by both low and higher strengths of stimulation were reduced during micturition. The sample of PSPs evoked by hindlimb cutaneous afferents in hindlimb motoneurons could also be attenuated during micturition. In contrast, monosynaptic EPSPs evoked by stimulation of low threshold muscle afferents recorded in eight hindlimb motoneurons were unchanged.

As discussed in the introductory section, suppression of EUS activity facilitates cfficient bladder cmptying during micturition. In the present study it was possible to evoke orthodromic action potentials that failed when the motoneuron membrane hyperpolarized during micturition (see also Shimoda et al., 1992). Hyperpolarization of EUS motoneurons decreases the likelihood of EPSPs reaching threshold for an action potential, and thus, the EUS muscle is less likely to be active during micturition. This hyperpolarization is produced by a micturitionrelated inhibitory input restricted to EUS motoneurons (see Results and Fedirchuk and Shefchyk, 1993). Since micturition caused a reduction of excitatory perineal reflexes to both EUS and EAS motoneurons in this study, it seems probable that preferential inhibition of EUS motoneurons during micturition can account for differences in activity patterns of these two motoneuron populations.

In addition to displacement of the EUS motoneuron membranc potential away from the threshold for cvoking action potentials, a membrane conductance increase associated with this postsynaptic inhibition (Fedirchuk and Shefchyk, 1993) could also decrease the amplitude of the EPSPs. Even though EAS and hindlimb motoneurons did not hyperpolarize during micturition, it is possible that a membrane conductance increase could have occurred and functioned to decrease the PSP amplitudes. However, in some hindlimb motoneurons, reductions in perineal-evoked EPSPs occurred during micturition without a change in monosynaptic EPSPs indicating that no membrane conductance change occurred (see similar example in Floeter et al., 1993). This observation implies that PSP reductions observed in these hindlimb motoneurons during micturition were due solely to premotoneuronal mechanisms.

One premotoneuronal mechanism that could contribute to widespread suppression of SPud and SPeri pathways is primary afferent depolarization (for review, see Rudomin, 1990). Onc can hypothesize that perineal afferents are the target of micturition-related PAD that results in decreased transmission from these afferents to interneurons interposed in pathways to EUS, EAS and hindlimb motoneurons. Recently, Angel et al. (in press) demonstrated that fewer than half of SPud afferents examined depolarized during micturition, and therefore it seems unlikely that PAD alone can account for the strong attenuation of all perineal reflexes examined in the present study. The possibility that other cutaneous primary afferents receive PAD during micturition remains to be examined. Interneurons have been described that mediate both PAD of muscle afferents and postsynaptic inhibition of hindlimb motoneurons (Solodkin et al., 1984). An attractive hypothesis is that similar interneurons exist in the micturition system and that these interneurons mediate both PAD of some perineal afferents and postsynaptic inhibition of EUS motoneurons. In addition, micturition-related removal of tonic PAD of some afferents (see Angel et al., in press) might account for the three examples where perineal-evoked EPSPs increased in amplitude during micturition (one EUS motoneuron, two PBSt motoneurons).

Another premotoneuronal mechanism that could contribute to the suppression of reflexes during micturition is the inhibition of interneurons interposed between perineal primary afferents and motoneurons. While a small proportion of perineal reflex pathways to motoneurons may be disynaptic, the majority of connections are at least trisynaptic (see latencies in Results and Fedirchuk et al., 1992a). Inhibition of first order interneurons shared by various perineal reflex pathways would be a convenient mechanism for suppressing various reflex pathways activated from perineal afferents. However, the possibility that other interneurons later in the various pathways are inhibited during micturition cannot be excluded at this time.

EUS motoneurons do not receive strong inputs from hindlimb cutaneous afferents in the decerebrate cat and thus modification of this weak input might not be crucial for successful micturition. The functions of perineal reflexes to hindlimb motoneurons and reduction of these reflexes seen during micturition are not known at this time. The reduction of both EPSPs and IPSPs evoked by hindlimb cutaneous afferents in hindlimb motoneurons demonstrates suppression of a variety of cutaneous reflexes during micturition. One explanation for this observation is that some perineal and hindlimb cutaneous afferents may share spinal interneurons and these interneurons are targeted for inhibition during micturition. Thus, the reduction in hindlimb cutaneous actions may be a consequence of this convergence. In addition, the examples of increased perineal EPSP amplitudes during micturition demonstrates the ability for micturition to facilitate these reflexes occasionally (i.e., removal of tonic PAD as mentioned earlier, or facilitation of interneurons).

The micturition circuitry can exert strong suppressive actions on perineal cutaneous reflexes regardless of their ultimate target motoneuron populations. There is evidence that afferents activated by bladder distension produce a small gradual hyperpolarization of EUS motoneuron membrane potential prior to micturition (Shimoda et al., 1992; Fedirchuk and Shefchyk, 1993). However, only upon activation of the micturition circuitry is there a more prominent hyperpolarization of EUS motoneurons, the occurrence of PAD in perineal afferents (Angel et al., in press), and reductions of perineal-evoked EPSPs. The time course of EUS motoneuron hyperpolarization during micturition matches well with the period of suppression of perineal cutaneous reflexes. Both could be the result of parallel actions exerted by the micturition circuitry onto different neurons (i.e., PAD interneurons, inhibitory interneurons). The locations of first order interneurons activated by SPud and SPeri stimulation have been identified (Fedirchuk et al., 1992b; Jankowska and Riddel, 1993). Future studies will examine the convergence of hindlimb and perineal afferent pathways on to these interneurons and determine if they receive an inhibitory input during micturition.

\section{References}

Angel MJ, McCrea DA, Fyda D, Shefchyk SJ (in press) PAD of pudendal afferents evoked by micturition and stimulation of hindlimb and perineal afferents in the cat. J Physiol (Lond), in press. 
deGroat WC (1990) Central control of the lower urinary tract In: Neurobiology of incontinence, Vol 151 (Bock G, Whelan J, eds), pp 27-56. New York: Wiley.

Fedirchuk B, Shefchyk SJ (1993) Membrane potential changes in sphincter motoneurons during micturition in the decerebrate cat. $\mathbf{J}$ Neurosci 13:3090-3094.

Fedirchuk B, Song L, Shefchyk SJ (1991) Investigation of the motoneuronal and premotoneuronal mechanisms of pudendal reflex modulation during micturition in the cat. Soc Neurosci Abstr 17:408.1.

Fedirchuk B, Hochman S, Shefchyk SJ (1992a) An intracellular study of perineal and hindlimb afferent inputs onto pudendal motoneurons in the decerebrate cat. Exp Brain Res 89:511-516.

Fedirchuk B, Song L, Downie JW, Shefchyk SJ (1992b) Spinal distribution of extracellular field potentials generated by electrical stimulation of pudendal and perineal afferents in the cat. Exp Brain Res 89:517-520.

Floeter MK, Sholomenko GN, Gossard JP, Burke RE (1993) Disynaptic excitation from the medial longitudinal fasciculus to lumbosacral motoneurons: modulation by repetitive activation, descending pathways, and locomotion. Exp Brain Res 92:407-419.

Garry RC, Roberts TDM, Todd JK (1959) Reflexes involving the external urethral sphincter in the cat. J Physiol (Lond) 149:653-665.
Jankowska E, Riddell JS (1993) A relay for input from group II muscle afferents in sacral segments of the cat spinal cord. J Physiol (Lond) 465:561-580.

Rampal G, Mignard P (1975) Organization of the nervous control of the urethral sphincter. Pfluegers Arch 353:21-31.

Rudomin P (1990) Presynaptic control of synaptic effectiveness of muscle spindle and tendon organ afferents in the mammalian spinal cord. In: The segmental motor system (Binder MD, Mendell LM, eds), pp 349-380.

Sethi RK, Bauer SB, Dyro FM, Krarup C (1989) Modulation of the bulbocavernosus reflex during voiding: loss of inhibition in upper motor neuron lesions. Muscle Nerve 12:892-897.

Shimoda N, Takakusaki K, Nishizawa O, Tsuchida S, Mori S (1992) The changes in the activity of pudendal motoneurons in relation to reflex micturition evoked in decerebrate cats. Neurosci Lett 135:175178 .

Solodkin M, Jimenez I, Rudomin P (1984) Identification of common interneurons mediating pre- and postsynaptic inhibition in the cat spinal cord. Science 224:1453-1456. 